\title{
Loss of GATA4 causes ectopic pancreas in the stomach
}

Running title: Pancreatic heterotopia in Gata4-deficient mice

Authors: Elisa Rodríguez-Seguel ${ }^{1 \S}$, Laura Villamayor ${ }^{1 \S}$, Noelia Arroyo ${ }^{1}$, Mónica Pérez $^{3}$, Francisco X. Real ${ }^{3,4,5}$, Franz Martín ${ }^{1,2}$, David A. Cano ${ }^{6}$ and Anabel Rojas ${ }^{1,2^{*}}$

\section{Affiliations:}

${ }^{1}$ Centro Andaluz de Biología Molecular y Medicina Regenerativa-CABIMER, Universidad Pablo de Olavide, Universidad de Sevilla, Consejo Superior de Investigaciones Científicas (CSIC), Seville, Spain

${ }^{2}$ Centro de Investigación Biomédica en Red de Diabetes y Enfermedades Metabólicas Asociadas (CIBERDEM), Madrid, Spain

${ }^{3}$ Centro Nacional de Investigaciones Oncológicas (CNIO), Madrid, Spain

${ }^{4}$ CIBERONC, Spain

${ }^{5}$ Departament de Ciències Experimentals i de la Salut, Universitat Pompeu Fabra, Barcelona, Spain

${ }^{6}$ Instituto de Biomedicina de Sevilla (IBIS), Hospital Universitario Virgen del Rocío/CSIC/Universidad de Sevilla, Seville, Spain

*Corresponding author

Avda. Américo Vespucio s/n.

Parque Científico Isla de la Cartuja

41092 Sevilla, Spain

E-mail: anabel.rojas@cabimer.es

Phone: (+34) 954467427

FAX: (+34) 954461664

$\S$ : These authors contributed equally to this study.

The authors have declared that no conflict of interest exists. 


\begin{abstract}
Pancreatic heterotopia is defined as pancreatic tissue outside its normal location in the body and anatomically separated from the pancreas. In this work we have analyzed the stomach glandular epithelium of Gata4floxfllox; Pdxl-Cre mice (Gata4KO mice). We found that Gata4KO glandular epithelium display an atypical morphology similar to the cornified squamous epithelium and exhibit upregulation of forestomach markers. The developing gastric units fail to form properly and the glandular epithelial cells do not express markers of gastric gland in the absence of GATA4. Interestingly, the developing glands of Gata4KO stomach express pancreatic cells markers. Furthermore, a mass of pancreatic tissue located in the subserosa of Gata4KO stomach is observed at adult stages. Heterotopic pancreas found in Gata4-deficient mice contains all three pancreatic cell lineages, ductal, acinar and endocrine cells. Moreover, Gata4 expression is downregulated in ectopic pancreatic tissue of some human biopsy samples.
\end{abstract}

Key words: GATA4 transcription factor, pancreatic heterotopia, stomach development, glandular stomach, pancreas. 


\section{Introduction}

Cell fate allocation during embryonic development is controlled by complex developmental programs that involve a network of signaling pathways and transcription factors. Deregulation of these developmental programs, including defects in organ patterning, morphogenesis or cell differentiation, might cause the formation of organs at ectopic locations.

Heterotopic pancreas is defined as pancreatic tissue outside its usual anatomical location but without anatomic or vascular connection to the normal pancreas. Pancreatic heterotopia is rare. In humans, ectopic pancreatic tissue is mainly found in the distal stomach and duodenum and at lower frequency in the esophagus, ileum and biliary tree [1]. Though mostly asymptomatic, this condition might result in pancreatitis, ulcerations, and gastric obstruction and, in rare cases, malignant degeneration [2]. The precise pathogenic mechanism of ectopic pancreas in human is poorly understood. Given the proximity of the pancreas to the stomach and duodenum along the primitive foregut, it has been proposed the misplacement of pancreatic cells into the developing gastrointestinal system as a hypothesis for pancreatic heterotopia [1]. An alternative hypothesis implies a metaplastic process in which ectopic pancreatic tissue would arise from other endodermal areas different from the pancreatic endoderm that undergo pancreatic metaplasia migrating to the submucosa of the gut during embryogenesis [3]. A third theory is based on the existence of totipotent cells lining the endoderm that differentiate into pancreatic tissue [4]. 
Several studies in mice have directly associated the formation of ectopic pancreas with defects in embryonic signaling pathways important for pancreas organogenesis. Thus, loss of Hes1, a Notch signaling transducer, leads to heterotopic pancreas in the stomach, duodenum and common biliary duct $[5,6]$. This seems to be due to defects in morphogenesis of the dorsal pancreas primordium which ingresses into the stomach domain during embryonic development [7]. Inhibition of Hedgehog signaling in the foregut also induces pancreatic heterotopia in stomach and duodenum $[8,9]$. Finally, genetic misexpression of the basic helix-loop-helix transcription factors Ptfla in the Pdxl expression domain of the foregut endoderm causes conversion of glandular stomach, anterior duodenum and the extra-hepatic biliary system into pancreas highlighting the critical role of this transcription factor in defining pancreas allocation in the foregut endoderm [10-12].

In the mouse, the stomach is divided into the forestomach (which it is not found in humans) that comprises a stratified squamous epithelium and the hindstomach or glandular stomach. The glandular stomach is composed of columnar epithelial cells and can be further divided into the corpus and the most posterior region, the antrum [13-15]. The stomach derives from the foregut endoderm, which also gives rise to pancreas among other endodermal-derived organs. Although far from being completely understood, the specification of the embryonic stomach is regulated by a number of signaling pathways (such as WNT, SHH and BMP) and transcriptions factors [14]. The regionalization of the gut endoderm is achieved in part by restricting the expression of transcription factors to particular domains. Thus, the foregut endoderm expresses SOX2 while the posterior region or hindgut expresses CDX2 [13-15]. Once the gastric identity is specified, the stomach undergoes further regionalization, a process that also involves 
selective expression of specific transcription factors in these areas. For example, $P d x l$ is expressed in the foregut region that gives rise to antrum and pylorus (in addition to pancreas, anterior duodenum and proximal extrahepatic biliary system) [16]. By contrast, Sox2 expression is maintained at much higher levels in the forestomach [17].

GATA4 is a member of the GATA zinc-finger transcription factor family that control specification, differentiation and proliferation of various mesoderm- and endoderm- derived cell types [18-22]. In the fetal stomach, Gata4 is expressed in the epithelium of the hindstomach and duodenum, and in adult stomach it is localized in pit, neck and parietal glandular epithelial cells [23-26] suggesting a role in glandular stomach formation. Indeed, studies using chimeric embryos, to circumvent the early lethality of Gata4 null mice [27-29] lends support to this notion. Gata4-null stomach cells fail to express markers associated with differentiation of gastric epithelial cell lineages [25]. However, it remains unclear whether these defects in glandular stomach cell differentiation reflect a cell-autonomous role of GATA4.

GATA4 has been shown to control cell identity during embryonic development by activating or repressing key regulators of endodermal fates. Thus, mice lacking GATA4 in the jejunum lost jejunal identity and fates into ileum lineage [30]. Interestingly, simultaneous inactivation of GATA4 and GATA6 results in dorsal and ventral pancreatic progenitors acquiring stomach and intestinal and lineages, respectively [31]. GATA4 is a key player in pancreas organogenesis. Mutations in GATA4 result in severe hypoplasia or complete pancreatic agenesis in humans [32]. In stark contrast, conditional inactivation of Gata4 in pancreatic progenitors does not have any apparent effect on pancreas formation in mice. However, mice with simultaneous 
inactivation of Gata4 and Gata6 fail to develop pancreas and died shortly after birth with hyperglycemia $[33,34]$ indicating functional redundancy of these transcription factor in mouse development.

In this work we have analyzed the stomach glandular epithelium of Gata4 floxflox; Pdxl-Cre mice (Gata4KO mice, hereafter). We found that Gata4KO glandular epithelium display an atypical morphology similar to the cornified squamous epithelium. The developing gastric units fail to form properly and the glandular epithelial cells do not express markers of gastric gland in the absence of GATA4. Interestingly, the developing glands of Gata4KO stomach express pancreatic cells markers and a mass of pancreatic tissue located in the subserosa of Gata4KO stomach is observed at adult stages. Heterotopic pancreas found in Gata4-deficient mice contains all three pancreatic cell lineages, ductal, acinar and endocrine cells. Analysis of ectopic pancreas in human biopsies revealed a downregulation of Gata4 expression in some of the samples analyzed. 


\section{MATERIAL AND METHODS}

\section{Mice}

Gata4 floxfllox, Pdxl-Cre, ROSA26ReYFP and Ptfla-Cre mice and the strategies for genotyping have been previously described [33,35-38]. All experiments using animals complied with institutional guidelines, and were reviewed and approved by the Institutional Animal care and Use Committee (IACUC) of the University of Sevilla, Spain or the Instituto de Salud Carlos III and Comunidad Autónoma de Madrid.

\section{Histology, Immunohistochemistry and Immunofluorescence.}

Dissected pancreata collected at adult stages were fixed in 4\% paraformaldehyde in phosphate-buffered saline (PBS) overnight at $4^{\circ} \mathrm{C}$, dehydrated with ethanol and xylene, and embedded in paraffin. Histological analyses were performed as previously described $[39,40]$. The following primary antibodies were used at the indicated dilution: mouse anti-GATA4 (1:100 Santa Cruz, Sc-25310); rabbit anti-Gastrin (1:50 Abcam, ab8492); mouse anti-alpha smooth muscle actin (1:300, Sigma-Aldrich, A5228); mouse anti-Hydrogen Potassium ATPase Beta (1:2000 Abcam, ab2866), rabbit anti-Sox2 (1:500 Abcam 97959), goat anti-GFP (1:200 Abcam, ab6673); guinea pig anti-Pdx1 (1:200 Abcam, ab47308); mouse anti-insulin (1:500 Sigma-Aldrich, I2018); mouse anti-E-Cadherin $(1: 100$, BD Bioscience, 61018); rabbit anti-Ptf1a (1:2000, Beta Cell Biology Consortium, AB2153); mouse anti-amylase (1:200 Santa Cruz, Sc-46657); mouse anti-MUC5AC (1:200 Thermo Scientific, MS-145-P); mouse anti-Glucagon (1:200, Sigma-Aldrich, G2654); rabbit anti-MafA (1:50, Bethyl laboratories, A300611A); rabbit anti-Glut2 (1:400, Millipore, 07-1402); rabbit anti- HNF1 $\beta$ (1:100, Santa Cruz Sc-8986), Biotinylated Dolichos biflorus Agglutinin (DBA) (1:300; Vector Laboratories, B-1035), rabbit anti-cytokeratin 14 (1:200, Abcam, ab15461); rabbit antiTrp63 (1:500, Abcam, ab53039). All pictures from immunofluorescence and 
immunohistochemistry shown are representative of at least 6 independent samples of the indicated genotype.

\section{Human Samples}

Gastric biopsies were obtained upon obtaining an informed consent from each patient. The study protocol was approved by the ethics committee of the Hospital Universitario Virgen del Rocío (HUVR), Seville and the study was conducted according to the principles of the Declaration of Helsinki. Immunofluorescence for GATA4 was performed on $4 \mu \mathrm{m}$ paraffin sections of human stomach from twenty-four patients. All samples analyzed contained pancreatic tissue located in the subserosa layer of the stomach. Eighteen samples out of the twenty-four also contained glandular stomach or proximal duodenum. 


\section{Results}

Morphological and cell differentiation defects in glandular stomach in the absence of Gata4

To investigate the role of GATA4 on the formation of the glandular stomach, mice with a conditional (flox) allele of Gata4 (Gata4floxflox $)$ were crossed with mice expressing the Cre recombinase under the control of the pancreatic and duodenal homeobox gene $1(P d x l)$ promoter (Pdxl-Cre mice) [36]. PDX1 is expressed in all pancreatic progenitors but is also expressed in the caudal region of the developing stomach as well as the duodenum, where it overlaps with GATA4 (Fig. 1A-C). To monitor Cre recombinase activity, Pdxl-Cre and Gata4floxflox; Pdxl-Cre mice were crossed to a ROSA26ReYFP reporter strain [37]. Immunofluorescence analyses of E17.5 embryos revealed robust $e Y F P$ expression in the antrum of both $P d x 1-C r e$; ROSA26ReYFP (Figure 1D, F) and Gata4tloxflox; Pdx1-Cre; ROSA26eEYFP embryos (Figure 1E, G). Scattered eYFP-positive cells were also observed in the corpus region along the greater curvature of the stomach in both mouse strains (Figure 1D, E, H, I). In agreement with this $C r e$ expression pattern, efficient GATA4 loss was observed in the antrum of Gata4 $4^{\text {floxflox }}$; Pdxl-Cre; ROSA26ReYFP embryos while a mosaic pattern of GATA4 protein is observed in the corpus (Fig. 1G, I). A similar result was obtained by immunohistochemistry analyses in Gataffloxflox; Pdxl-Cre (hereafter, Gata4KO mice) and control embryos (Gatafflox/+ $;$ Pdxl-Cre or Gata4 $4^{\text {floxflox }}$ (Fig. 1J-M).

We next analyzed whether the lack of GATA4 affects stomach development by analyzing the stomach epithelium at various embryonic stages. No major morphological abnormalities were observed at embryonic day (E) 13.5 and E15.5 (Supplemental Fig. 1). However, by E17.5, Gata4KO embryos failed to form the characteristic developing 
gastric units of normal stomach (Supplemental Figure 1). In newborn mice, Gata4KO stomach lacked gastric glands and the epithelium appeared mostly flattened (Fig. 2B, D) in contrast to control stomach in which invaginations of the gastric epithelium were clearly observed (Fig. 2A, C). The stomach of adult (2-3-month-old) control mice displayed a well-defined and highly organized glandular epithelium (Fig. 2E, G). In stark contrast, Gata4-deficient antrum showed an atypical morphology with appearance of a cornified squamous epithelium (Fig. 2F, H). To determine whether glandular cell differentiation was impaired in Gata4KO stomach, we analyzed several markers of differentiated gastric cells. At E17.5, parietal cells expressing the gastric hydrogen potassium ATPase ( $\mathrm{HK}^{+}$-ATPase) in the corpus and gastrin-expressing enteroendocrine cells in the antrum were easily observed in the developing glands of control mice (Supplemental Fig.1). However, only few $\mathrm{HK}^{+}$-ATPase-expressing cells were found in the corpus of Gata4 $\mathrm{KO}$ embryos, likely due to mosaic Cre activity observed in this region of the stomach (Supplemental Fig.1). No gastrin-expressing cells were observed in the antrum epithelium of the Gata4KO embryos (Supplemental Figure 1). At both P1 and adult stomach, well-differentiated parietal cells (positive for $\mathrm{HK}^{+}$-ATPase) (Fig. 2I, K), mucus-producing cells (positive for MUC5AC) (Fig. 2M, O) and G-enteroendocrine cells (positive for Gastrin) were found in control mice (Fig. 2Q, S). In stark contrast, the expression of HK+-ATPase, MUC5AC and Gastrin were dramatically reduced in the glandular epithelium of Gata4 knockout mice (Fig. 2J, L, N, P, R, T). These results indicate that GATA4 plays a critical role in the differentiation of the gastric epithelium.

\section{Upregulation of squamous epithelial markers in the corpus and antrum of Gata4KO}

\section{mice}


The cornified appearance of the corpus and antrum of Gata4-deficient mice, prompted us to investigate the expression of squamous epithelial markers. Cytokeratin 14 and the transcription factor TRP63 are expressed in forestomach but not in corpus and antrum. As expected, immunofluorescence analyses for these squamous epithelial markers revealed a clear boundary between the forestomach and corpus in newborn (Fig. 3A, A', C, C'). and adult control mice (Fig. 3G, G' I, I'). However, this boundary was not apparent in Gata4KO stomach and both the antrum and corpus showed a marked increase in cytokeratin 14 (Fig. 3B, B', B', H, H') and TRP63 (Fig. 3D, D', D', J, J') immunostaining. The gastric SRY-related HMG transcription factor SOX2 was strongly expressed in the forestomach whereas is much lower expressed in both corpus and antrum of newborn (Fig. 3E, E'), and adult control stomach (Fig. 3K, K'), as previously reported [13]. However, a dramatic upregulation of SOX2 expression was observed in the corpus and antrum of newborn (Fig. 3F, F', F'') and adult Gata4KO mice (Fig. 3L, L'). Altogether, these results demonstrate that GATA4 is required to maintain glandular stomach identity.

\section{Ectopic pancreas in Gata4-deficient mice}

Histological analyses of embryonic stomach at E17.5 revealed an ingression of the flattened epithelium of embryonic Gata4KO antrum into the subserosa layer of the stomach. Remarkably, the invaginated gastric epithelium formed a dilated duct structure adjacent to clusters of cells with pancreatic acinar morphology (Fig. 4D). By contrast, control stomach displayed normal developing unit glands (Fig. 4A). At P1 and in adult stomach of Gata4KO mice, this ectopic pancreatic tissue expanded and it was welldistinguishable by hematoxylin and eosin staining (Fig. 4G, J, M). The penetrance of the ectopic pancreas phenotype in all analyzed neonates and adult Gata4KO mice was 
$100 \%$ (20 neonates and 17 adult mice in total), while it was never observed in control mice (>50 analyzed mice). Gata4 floxflox; Pdx1-Cre ROSA26R-eYFP mice were used to better visualize the ectopic pancreatic tissue. In control Pdxl-Cre ROSA26R-eYFP embryonic stomach, eYFP-labelled cells were only observed in the developing glands (Fig. 4B). However, eYFP-labelled Gata4 knockout cells in the subserosa layer of the stomach appeared continuous with the gastric epithelium, discontinuing the submucosal and muscular layer, as revealed by smooth muscle $\alpha$-actin immunostaining (Fig. 4E, H, K). Interestingly, cells from PDX1-positive ducts located in the subserosa layer of the stomach (Fig. 4F, L) clustered into acinar-like structures that expressed amylase (Fig. 4F, I, N) and the acinar transcription factor PTF1A (Fig. 4O). No immunostaining for amylase was observed in the control stomach (Fig. 4C). Remarkably, endocrine cells expressing insulin and glucagon were also detected in the ectopic pancreas in Gata4KO stomach (Fig. 4P). The distribution of endocrine cells in the ectopic pancreas resembled murine pancreatic islets, with a core of insulin-positive cells surrounded by glucagonpositive cells (Fig. 4P). Moreover, insulin-producing cells expressed the mature $\beta$ cell markers MAFA (Fig. 4Q) and the glucose sensor GLUT2 (Fig. 4R).

\section{Gata4-deficient gastric epithelium expresses pancreatic markers}

Ectopic pancreas has been associated to impaired Notch signaling in mice [5-7]. We examined the expression of Hes 1, a mediator of Notch signaling, and its downstream target Ngn3 in E13.5 and E15.5 Gata4KO pancreas and stomach. Quantitative RT-PCR analysis showed similar Hes1 and Ngn3 expression levels in Gata4KO pancreas compared to control pancreas at both stages (Supplemental Figure 2). Similarly, Hes 1 and Ngn3 expression was not altered in the absence of GATA4 activity in the stomach (Supplemental Figure 2). Moreover, in contrast to what it is 
observed in Hes1 mutant embryonic pancreata, the dorsal pancreas of Gata4 KO mice displayed normal morphology and the pancreatic epithelium, labeled by E-cadherin, did not contact the stomach epithelium, (Supplemental Figure 2). These results indicate that the formation of ectopic pancreas in Gata4KO embryos is not due to decreased Notch signaling. Inhibition of Hedgehog signaling in the early foregut endoderm has also been associated with pancreatic heterotopia in stomach [8,9]. However, the gastric epithelium in the Gata4KO antrum showed a marked upregulation of Shh expression (Supplemental Figure 2), and therefore it does not support a role for loss of Hedgehog signaling in pancreatic heterotopia of Gata4 $\mathrm{KO}$ mice. Indeed, our results are in agreement with a previous study showing that GATA4 attenuates Shh expression in the distal stomach [25].

To determine whether loss of GATA4 specifically in pancreatic progenitors leads to the formation of ectopic pancreas in a wildtype stomach, we crossed Gata4 floxed mice with Ptfla-Cre mice [38]. Ptfla-Cre is active in pancreatic cells from E9.5 onwards although it is later restricted to the acinar cell lineage. However, it is not expressed in the stomach. Accordingly, strong GATA4 expression was observed in the glandular stomach of Gata4floxflox; Ptfla-Cre mice (Supplemental Figure 3).

Histological and immunohistochemical analyses revealed well-differentiated gastric unit glands in Gata4 floxfflox; Ptfla-Cre mice (Supplemental Figure 3). Furthermore, no ectopic pancreatic tissue was observed in any of the Gata4floxflox; Ptfla-Cre conditional knockout mice analyzed $(n=10)$. These data indicate that the absence of GATA4 in the pancreatic compartment does not induce the formation of ectopic pancreatic tissue in the stomach. The morphological analysis of the developing gastric antrum of Gataffloxflox; Pdxl-Cre embryos suggests that the ectopic pancreas originates directly 
from the glandular epithelium rather than from the migration of pancreatic cells into the stomach antrum during development (Figure 4E, H). To test this hypothesis, we analyzed the gastric epithelium of Gata4-deficient embryos for expression of pancreatic markers. As expected, the developing gastric glands and proximal duodenum in control embryos strongly expressed $P d x l$ (Fig. 5A, C) but no insulin-positive endocrine cells were found. In stark contrast, scattered insulin-positive cells were observed in the glandular stomach and in the duodenum of the Gata4-deficient glandular epithelium (Fig. 5B, D). Moreover, an upregulation of the ductal marker HNF1 $\beta$ is observed in areas of Gata4 knockout glandular epithelium that invaginates into the subserosa layer, discontinuing the smooth muscle layer (Fig. 5F). Interestingly, the HNF1 $\beta$-positive cells strongly react with Dolichos biflorus agglutinin (DBA) (Fig. 5H) which selectively labels ductal cells in the pancreas [41]. No upregulation of HNF1 $\beta$ was observed in the glandular epithelium of control embryos (E, G).

\section{GATA4 is downregulated in human heterotopic pancreas}

To investigate the potential role of GATA4 in human pancreatic heterotopia, we compared its expression in samples of 24 patients containing ectopic pancreatic tissue and in normal pancreatic tissue. In 8 of the cases, strong GATA4 immunostaining was detected in the acinar cells of the ectopic pancreas, similar to the immunostaining observed in normal pancreas (Fig. 6B, G). However, 16 samples displayed low accumulation of GATA4 protein in ectopic pancreas compared to normal, orthotopic pancreas (Fig. 6L). Of note, all analyzed samples showed strong immunostaining for the acinar marker amylase serving as an internal control for adequate immunohistochemical analysis (Fig. 6E, J, O). Out of the 24 samples from patients containing heterotopic pancreas, 18 also contained glandular stomach/proximal duodenum, which allowed us 
to evaluate GATA4 expression in both tissues of the same patient. Remarkably, the samples with weak GATA4 immunostaining in the ectopic pancreas also displayed weak GATA4 immunostaining in the glandular stomach and proximal duodenum (Fig. $6 \mathrm{D}, \mathrm{I}, \mathrm{N})$. This result might suggest that, at least in some cases, GATA4 downregulation might be associated with formation of ectopic pancreas in humans. 


\section{Discussion}

Here we show that the transcription factor GATA4 is critical for the maintenance of cell identity in the hindstomach and the differentiation of glandular epithelial cells. The inactivation of Gata4 in the stomach using the Pdxl-Cre driver mouse strain, impairs glandular epithelium formation and leads to aberrant induction of forestomach markers in the glandular stomach. More remarkably, Gata4 deficiency causes ectopic pancreas in the subserosa layer of the stomach.

Our results provide support to previous studies that have suggested an important role for GATA4 in glandular stomach development [24,25]. GATA4 is expressed in the hindstomach but not in the forestomach during embryonic development. Studies on chimeric embryos have shown that Gata4-null cells contribute to the squamous gastric epithelium but not to the glandular epithelium. However, these studies could not distinguish whether GATA4 was necessary for gastric specification and/or differentiation. We used the Pdxl-Cre mouse line to inactivate Gata4, which targets the antral region precursors and, less efficiently in a mosaic fashion, the corpus. Therefore, inactivation of Gata4 in using Pdx1-Cre driver mice would occur only after these regions have been specified. Further studies inactivating Gata4 in the foregut epithelium would be necessary to establish the role of GATA4 in stomach specification. Although we could not properly examine the role of GATA4 in this process, our results demonstrate that GATA4 is necessary for glandular stomach cell differentiation. Thus, the expression of markers of parietal cells, enteroendocrine, and mucus-producing cells is dramatically reduced in the Gata4KO stomach. In agreement with our findings, it was shown in the above study that the scarce Gata4-null cells incorporated into the chimeric glandular stomach lacked expression of gastric markers. Interestingly, we found that 
Gata4KO embryos exhibit a transformation of the hindstomach from columnar to a stratified squamous type epithelial morphology. Notably, increased expression of forestomach epithelium markers including the transcription factor SOX2, cytokeratin 14 and TRP63 were observed in the antral and corpus region of Gata4KO stomach suggesting that loss of GATA4 might lead to anteriorization of the hindstomach. These data suggest that GATA4 maintains glandular stomach cell identity by repressing markers of the anterior stomach. This role is reminiscent of the role of GATA4 in regulating the jejunal-ileal boundary in the small intestine $[42,43]$. The exact mechanism by which GATA4 maintains hindstomach identity remains to be determined. However, loss of GATA4 results in aberrant Sox2 expression in the glandular stomach and SOX2 plays a key role in gastric epithelial differentiation. Indeed, misexpression of Sox 2 in the posterior region of the primitive gut induces a gastric-like phenotype in the intestine [44]. Thus, it is tempting to speculate whether GATA4 might directly repress Sox2 expression in glandular stomach.

Unexpectedly, Gata4KO mice exhibited pancreatic heterotopia in the stomach. Cells of all pancreatic lineages (acinar, endocrine and ductal) were found in Gata4KO stomach. Importantly, ectopic pancreas was only observed in the hindstomach or in the proximal duodenum. The molecular determinants of pancreatic heterotopia and the pathogenic mechanisms involved are not well known. Earlier studies suggest that pancreatic heterotopia in mice with mutations in Hesl was caused by transdifferentiation of the embryonic stomach to pancreatic tissue [6], but a recent study proposes that aberrant morphogenesis of the dorsal pancreas primordium is the underlying mechanism in Hes 1 mutant mice [7]. We did not observe changes in Hes 1 and Ngn3 (a HES1 target gene) expression in the dorsal pancreas of Gata4KO embryos, 
suggesting that the heterotopic pancreas observed in Gata4-deficient mice might be independent of Notch signaling.

Although we cannot conclusively identify the basis for the pancreatic heterotopia in Gata4KO mice, our observations provide evidence, albeit circumstantial, for a transcommitment of regions of the prospective glandular stomach to pancreatic lineage. The inactivation of Gata4 exclusively in the pancreatic progenitors using a highly pancreas-specific Cre mouse line (Ptfla-Cre) did not lead to the formation of ectopic pancreas, suggesting that the absence of GATA4 in the stomach domain is selectively required for this embryonic disorder. The presence of scattered insulin positive cells and the upregulation of HNF1 $\beta$ ductal marker in the developing unit glands of Gata4-deficient mice might suggest that endodermal tissue migrates to the submucosa during embryogenesis transforming into pancreatic tissue. However, this theory fails to explain why full tissue conversion is not observed in the entire $P d x l$ positive domain of the glandular stomach. Further studies will be required to determine the exact mechanism. Nevertheless, our findings point to an important role of GATA4 in establishing cell lineages during foregut embryonic development. Indeed, a recent study supports a role for GATA factors in the foregut patterning. Simultaneous inactivation of Gata4 and Gata6 results in pancreatic agenesis [33,34]. Further studies in the embryonic pancreatic rudiments of these double mutants have shown a cell fate switch in dorsal and ventral pancreas to stomach and intestine, respectively [31]. However, individual Gata4KO embryos were not analyzed in this study.

In humans, pancreatic heterotopia is most commonly observed in stomach and intestine and, more rarely, in esophagus, biliary tract and spleen. We examined GATA4 
expression in a series of human gastric biopsies with pancreatic heterotopia. Although far from conclusive, a substantial number of these samples showed decreased GATA4 expression in the ectopic pancreas suggesting that GATA4 might be associated with pancreatic heterotopia in humans as well. Interestingly, decreased GATA4 expression in ectopic pancreas correlates with decreased GATA4 expression in the glandular stomach and proximal duodenum. These results, together with our observations inactivating Gata4 using the Ptfla-Cre line, support the idea that specific loss of GATA4 in the stomach/duodenum causes the formation of ectopic pancreas.

Our findings raise the question as to whether mutations in GATA4 (or other genes regulating GATA4 expression) might be linked to pancreatic heterotopia in humans. Patients with GATA4 mutations have been reported. Indeed, a recent study has reported that mutations in GATA4 are a cause of neonatal or childhood-onset diabetes [32]. However, no analysis of the stomach in these patients has been described and deeper imaging studies of these patients might be warranted.

\section{Acknowledgements:}

We thank Antonio Cárdenas and Ana Belén Hitos for their technical assistance. E. R-S. was supported by a "Juan de la Cierva” posdoctoral fellowship from Spanish Ministry of Economy and Competitiveness (IJCI-2014-19251). L.V. was supported by a contract from Spanish Ministry of Economy and Competitiveness (RYC-2013-14533). This work was supported by grants from the Spanish Ministry of Economy and Competitiveness BFU2017-82497-P to AR, ISCIII-Subdirección General de Evaluación y Fomento de la Investigación co-funded with Fondos FEDER, PI16/00175 to D.A.C., 
the Andalusian Ministry of Science and Innovation CTS-7478 to D.A.C) and grant SAF2015-70553-R to FXR. CNIO is supported by the "Ministerio de Ciencia, Innovación y Universidades" as a Centro de Excelencia Severo Ochoa (SEV-20150510). This research has been conducted using samples from the HUVR-IBiS Biobank (Andalusian Public Health System Biobank and ISCIII-Red de Biobancos PT17/0015/0041). The authors would like to thank Carolina Castilla and Fernando Carmona (HUVR-IBiS Biobank) for their outstanding technical help.

\section{Author contributions}

E.R.S., L.V., N, A., M.P. conceived and carried out experiments. F.M, F.X.R., D.A.C, and A.R participated in the design of the study, conceived and analyzed the data. D.A.C. and A.R. wrote the manuscript. All authors reviewed the paper and had final approval of the submitted manuscript. 


\section{References}

1. Kung JW, Brown A, Kruskal JB, et al. Heterotopic pancreas: typical and atypical imaging findings. Clin Radiol 2010; 65: 403-407.

2. Cazacu IM, Luzuriaga Chavez AA, Nogueras Gonzalez GM, et al. Malignant Transformation of Ectopic Pancreas. Dig Dis Sci 2019; 64: 655-668.

3. Park E, Kim H, Jung KW, et al. Heterotopic pancreas in omphalomesenteric duct remnant results in persistent umbilical discharge. Korean J Pathol 2014; 48: 323-326.

4. Stock C, Keutgen XM, Pisapia D, et al. Heterotopic pancreatic neoplasm presenting as an obstructing mass at the fourth portion of the duodenum. JOP 2011; 12: 241-243.

5. Sumazaki R, Shiojiri N, Isoyama S, et al. Conversion of biliary system to pancreatic tissue in Hes1-deficient mice. Nat Genet 2004; 36: 83-87.

6. Fukuda A, Kawaguchi Y, Furuyama K, et al. Ectopic pancreas formation in Hes 1 -knockout mice reveals plasticity of endodermal progenitors of the gut, bile duct, and pancreas. J Clin Invest 2006; 116: 1484-1493.

7. Jorgensen MC, de Lichtenberg KH, Collin CA, et al. Neurog3-dependent pancreas dysgenesis causes ectopic pancreas in Hes1 mutant mice. Development $2018 ; 145$.

8. Hebrok M, Kim SK, Melton DA. Notochord repression of endodermal Sonic hedgehog permits pancreas development. Genes Dev 1998; 12: 1705-1713.

9. Kawahira H, Ma NH, Tzanakakis ES, et al. Combined activities of hedgehog signaling inhibitors regulate pancreas development. Development 2003; 130: 4871-4879.

10. Afelik S, Chen Y, Pieler T. Combined ectopic expression of Pdx 1 and Ptf1a/p48 results in the stable conversion of posterior endoderm into endocrine and exocrine pancreatic tissue. Genes Dev 2006; 20: 1441-1446.

11. Jarikji ZH, Vanamala S, Beck CW, et al. Differential ability of Ptfla and PtflaVP16 to convert stomach, duodenum and liver to pancreas. Dev Biol 2007; 304: 786-799.

12. Willet SG, Hale MA, Grapin-Botton A, et al. Dominant and context-specific control of endodermal organ allocation by Ptfla. Development 2014; 141: 43854394.

13. Kim TH, Shivdasani RA. Stomach development, stem cells and disease. Development 2016; 143: 554-565.

14. McCracken KW, Wells JM. Mechanisms of embryonic stomach development. Semin Cell Dev Biol 2017; 66: 36-42.

15. Willet SG, Mills JC. Stomach Organ and Cell Lineage Differentiation: from Embryogenesis to Adult Homeostasis. Cell Mol Gastroenterol Hepatol 2016; 2: 546-559.

16. Offield MF, Jetton TL, Labosky PA, et al. PDX-1 is required for pancreatic outgrowth and differentiation of the rostral duodenum. Development 1996; 122: 983-995.

17. Que J, Luo X, Schwartz RJ, et al. Multiple roles for Sox2 in the developing and adult mouse trachea. Development 2009; 136: 1899-1907.

18. Arceci RJ, King AA, Simon MC, et al. Mouse GATA-4: a retinoic acidinducible GATA-binding transcription factor expressed in endodermally derived tissues and heart. Mol Cell Biol 1993; 13: 2235-2246.

19. Bossard P, Zaret KS. GATA transcription factors as potentiators of gut endoderm differentiation. Development 1998; 125: 4909-4917. 
20. Rojas A, De Val S, Heidt AB, et al. Gata4 expression in lateral mesoderm is downstream of BMP4 and is activated directly by Forkhead and GATA transcription factors through a distal enhancer element. Development 2005; 132: 3405-3417.

21. Rojas A, Kong SW, Agarwal P, et al. GATA4 is a direct transcriptional activator of cyclin D2 and Cdk4 and is required for cardiomyocyte proliferation in anterior heart field-derived myocardium. Mol Cell Biol 2008; 28: 5420-5431.

22. Rojas A, Schachterle W, Xu SM, et al. An endoderm-specific transcriptional enhancer from the mouse Gata4 gene requires GATA and homeodomain protein-binding sites for function in vivo. Dev Dyn 2009; 238: 2588-2598.

23. Nemer G, Nemer M. Transcriptional activation of BMP-4 and regulation of mammalian organogenesis by GATA-4 and -6. Dev Biol 2003; 254: 131-148.

24. Jacobsen CM, Mannisto S, Porter-Tinge S, et al. GATA-4:FOG interactions regulate gastric epithelial development in the mouse. Dev Dyn 2005; 234: 355362.

25. Jacobsen CM, Narita N, Bielinska M, et al. Genetic mosaic analysis reveals that GATA-4 is required for proper differentiation of mouse gastric epithelium. Dev Biol 2002; 241: 34-46.

26. Jonckheere N, Vincent A, Franquet-Ansart H, et al. GATA-4/-6 and HNF-1/-4 families of transcription factors control the transcriptional regulation of the murine Muc5ac mucin during stomach development and in epithelial cancer cells. Biochim Biophys Acta 2012; 1819: 869-876.

27. Kuo CT, Morrisey EE, Anandappa R, et al. GATA4 transcription factor is required for ventral morphogenesis and heart tube formation. Genes Dev 1997; 11: $1048-1060$.

28. Molkentin JD, Lin Q, Duncan SA, et al. Requirement of the transcription factor GATA4 for heart tube formation and ventral morphogenesis. Genes Dev 1997; 11: 1061-1072.

29. Narita N, Bielinska M, Wilson DB. Wild-type endoderm abrogates the ventral developmental defects associated with GATA-4 deficiency in the mouse. Dev Biol 1997; 189: 270-274.

30. Battle MA, Bondow BJ, Iverson MA, et al. GATA4 is essential for jejunal function in mice. Gastroenterology 2008; 135: 1676-1686 e1671.

31. Xuan S, Sussel L. GATA4 and GATA6 regulate pancreatic endoderm identity through inhibition of hedgehog signaling. Development 2016; 143: 780-786.

32. Shaw-Smith C, De Franco E, Lango Allen H, et al. GATA4 mutations are a cause of neonatal and childhood-onset diabetes. Diabetes 2014; 63: 2888-2894.

33. Carrasco M, Delgado I, Soria B, et al. GATA4 and GATA6 control mouse pancreas organogenesis. J Clin Invest 122: 3504-3515.

34. Xuan S, Borok MJ, Decker KJ, et al. Pancreas-specific deletion of mouse Gata4 and Gata6 causes pancreatic agenesis. J Clin Invest 122: 3516-3528.

35. Watt AJ, Zhao R, Li J, et al. Development of the mammalian liver and ventral pancreas is dependent on GATA4. BMC Dev Biol 2007; 7: 37.

36. Hingorani SR, Petricoin EF, Maitra A, et al. Preinvasive and invasive ductal pancreatic cancer and its early detection in the mouse. Cancer Cell 2003; 4: 437450.

37. Srinivas $\mathrm{S}$, Watanabe T, Lin CS, et al. Cre reporter strains produced by targeted insertion of EYFP and ECFP into the ROSA26 locus. BMC Dev Biol 2001; 1: 4. 
38. Kawaguchi Y, Cooper B, Gannon M, et al. The role of the transcriptional regulator Ptfla in converting intestinal to pancreatic progenitors. Nat Genet 2002; 32: 128-134.

39. Delgado I, Carrasco M, Cano E, et al. GATA4 loss in the septum transversum mesenchyme promotes liver fibrosis in mice. Hepatology 2014; 59: 2358-2370.

40. Munoz-Bravo JL, Hidalgo-Figueroa M, Pascual A, et al. GDNF is required for neural colonization of the pancreas. Development 2013; 140: 3669-3679.

41. Kobayashi H, Spilde TL, Li Z, et al. Lectin as a marker for staining and purification of embryonic pancreatic epithelium. Biochem Biophys Res Commun 2002; 293: 691-697.

42. Bosse T, Piaseckyj CM, Burghard E, et al. Gata4 is essential for the maintenance of jejunal-ileal identities in the adult mouse small intestine. $\mathrm{Mol}$ Cell Biol 2006; 26: 9060-9070.

43. Thompson CA, Wojta K, Pulakanti K, et al. GATA4 Is Sufficient to Establish Jejunal Versus Ileal Identity in the Small Intestine. Cell Mol Gastroenterol Hepatol 2017; 3: 422-446.

44. Raghoebir L, Bakker ER, Mills JC, et al. SOX2 redirects the developmental fate of the intestinal epithelium toward a premature gastric phenotype. $\mathrm{J} \mathrm{Mol} \mathrm{Cell}$ Biol 2012; 4: 377-385. 


\section{Figure Legends}

Figure 1. Abnormal morphology of the glandular stomach in Gata4-deficient mice. Immunofluorescence analyses demonstrate overlapping expression of GATA4 and PDX1 in the antrum of wild-type embryos (A-C). Strong eYFP staining in the antrum (a) of Pdxl-Cre; Rosa26RYFP (Pdxl-Cre;eYFP) and Gata4floxflox; Pdxl-Cre; ROSA26ReYFP (G4KO;eYFP) embryos at E17.5. A mosaic pattern for eYFP is found in the greater curvature of the corpus $(\mathrm{c})(\mathrm{D}, \mathrm{E})$. Higher magnification of the respective antral region are shown in $\mathrm{F}$ and $\mathrm{G}$. Higher magnification of the respective corpus region are shown in $\mathrm{H}$ and I. Loss of Gata4 in the antrum of Gataffloxflox; Pdxl-Cre (G4KO) of E17.5 embryos (K) compared to Ctrl embryos (J). Strong GATA4 staining is observed in the corpus of E17.5 Ctrl embryos (L), whereas incomplete inactivation of Gata4 is detected in the corpus of G4KO embryos (M). Scale bars: (A-G): $100 \mu \mathrm{m}$; (HM): $25 \mu \mathrm{m}$.

\section{Figure 2. Defects in morphology and cell differentiation in Gata4-deficient gastric} epithelium.

Hematoxylin/Eosin staining of stomach tissue shows the antral epithelium of P1 Gata4deficient mice $(\mathrm{G} 4 \mathrm{KO})$ is flattened and glands are not properly formed $(\mathrm{B}, \mathrm{D})$, in contrast to control (Ctrl) antrum epithelium in which the developing unit glands are easily observed (A, C). In the adult, the stomach of control mice displays wellorganized gastric glands (E, G). G4KO antrum displays abnormal glandular morphology composed of epithelial cells with cornified appearance $(F, H)$. Inset in $\mathrm{H}$ shows higher magnification of the cornified gastric epithelium. 
Immunostaining analysis of the stomach of control $(\mathrm{Ctrl})$ and Gata4 $K O(\mathrm{G} 4 \mathrm{KO})$ at $\mathrm{P} 1$ and adult stages for the parietal cell marker, $\mathrm{HK}^{+}$-ATPase (I-L), the pit cell marker MUC5AC (M-P) and G cell marker gastrin (Q-T) reveals defects in glandular cell differentiation in Gata4KO (G4KO) mice. Inset in $\mathrm{S}$ shows higher magnification of

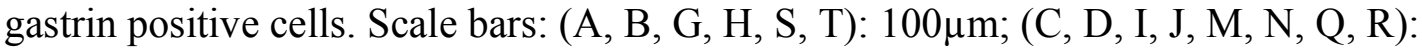
$25 \mu \mathrm{m} ;(\mathrm{E}, \mathrm{F}, \mathrm{K}, \mathrm{L}, \mathrm{O}, \mathrm{P}): 500 \mu \mathrm{m}$.

\section{Figure 3. GATA4 maintains stomach glandular cell identity}

Immunostaining for cytokeratin 14 revealed a clear junction between the forestomach and hindstomach in control (Ctrl) mice at both P1 (A, A') and adult stages (G, G'). Gata4KO mice exhibit upregulation of cytokeratin 14 expression in the corpus and antrum at P1 (B, B', B') and at adult ages (H, H'). GATA4 is exclusively expressed in the columnar epithelium of P1 (C, C', E, E') and adult control stomach (I, I', K, K'). TRP63 protein is restricted to the forestomach in control mice at P1 $\left(\mathrm{C}, \mathrm{C}^{\prime}\right)$ and in adult stages (I, I'). In Gata4KO mice, TRP63 immunostaining is observed in the antrum and corpus of neonate (D, D') and adult (J, J') mice. Sox2 is highly expressed in the forestomach of control neonate (E, E') and adult (K, K') mice. An upregulation of Sox2 expression is observed in the antrum and corpus of Gata4KO mice at P1 (F, F') and adult ages (L, L'). Note that cells that escape from Cre recombination in the corpus of Gata4KO mice are positive for GATA4 and negative for TRP63 (D') and SOX2 (F'). Counterstaining with DAPI (blue) was performed to visualize nuclei in immunofluorescence analyses. fs: forestomach; c: corpus, a: antrum. A' through F', are high magnification pictures from A through F, respectively. Scale bars: (A-F, G-L): $500 \mu \mathrm{m} ;\left(\mathrm{A}^{\prime}-\mathrm{F}^{\prime}, \mathrm{G}^{\prime}-\mathrm{L}^{\prime}\right): 100 \mu \mathrm{m}$. 


\section{Figure 4: Ectopic formation of pancreatic tissue in the distal stomach of Gata4-}

deficient mice. Histological and immunofluorescence analyses of the stomach of control (Ctrl) and Gata4 knockout (G4KO) E17.5 embryos (A-F), P1 neonates (G-I) and adult mice (J-R). Embryonic G4KO stomach tissue displays invagination of the antrum epithelium into the subserosa layer forming a duct-like structure (D), as observed by Hematoxylin/Eosin staining. Normal stomach morphology in control embryos (A). Hematoxylin/Eosin staining reveals well-distinguishable pancreatic tissue in the subserosa of Gata4KO stomach at P1 (G) and adult stages (J, M). eYFP-labelled cells in the ectopic pancreas of adult Gata4tloxflox; Pdxl-Cre ROSA26R-eYFP mice are continuous with the stomach epithelium and penetrate the submucosal and muscular layers of the antrum, marked by smooth $\alpha$-actin ( $\alpha$-SMA) immunoreactivity (E, H, K). Ectopic pancreas of Gata4KO contains well-differentiated acinar cells that express amylase (F, I, N). Ectopic pancreatic epithelium, marked by Ecadherin, show expression of the acinar marker PTF1A (O) . At adult stages, small clusters composed of endocrine insulin- and glucagon-expressing cells (P) are observed. Insulin-expressing cells express MAFA (Q). Insulin-expressing cells, marked by PDX1 immunoreactivity, also express the glucose transporter GLUT2 (R) . Counterstaining with DAPI was performed to visualize nuclei (blue) in immunofluorescence analyses. Scale bars: (A-N, P-R): $100 \mu \mathrm{m} .(\mathrm{O}): 25 \mu \mathrm{m}$.

\section{Figure 5: Pancreatic marker expression in the glandular stomach of Gata4KO}

embryos. Immunofluorescence analyses reveal the presence of insulin-positive cells in the developing glandular stomach (B) and proximal duodenum (D) of E17.5 Gata4 knockout (G4KO) embryos. No insulin positive cells are found in stomach or 
duodenum epithelium of E17.5 control embryos (A, C). Areas of the $G 4 K O$ glandular epithelium invaginating into the subserosa layer and discontinuing the smooth muscle layer exhibited upregulation of the ductal pancreatic marker $\operatorname{Hnf} 1 \beta(\mathrm{F})$, in parallel with increased reactivity for the ductal marker DBA lectin $(\mathrm{H})$. No immunostaining for HNF1 $1 \beta$ and DBA lectin is observed in control stomach (E, G). Counterstaining with DAPI was performed to visualize nuclei (blue). Arrowheads in B and D indicate insulin-producing cells. Scale bars: (A-F): $100 \mu \mathrm{m} ;(\mathrm{G}, \mathrm{H}): 25 \mu \mathrm{m}$.

\section{Figure 6: GATA4 expression in human heterotopic pancreas.}

Hematoxylin/eosin and immunofluorescence analyses of human gastric biopsies of control individuals (A-E) and patients with heterotopic pancreas (F-O). Eight (out of 24) biopsies of heterotopic pancreas display GATA4 protein levels in the ectopic pancreas (G) and in the glandular stomach (I) similar to those observed in normal pancreas (B) and in stomach of healthy patients (D). However, the remaining 16 biopsies exhibit a dramatic decrease in the accumulation of GATA4 protein in both the ectopic pancreas (L) and proximal duodenum (N). Of note, all patients analyzed show similar (and robust) levels of amylase protein in the pancreatic tissue (E, J, O). Scale bars: (A-D, F-I, $\mathrm{K}-\mathrm{N}): 100 \mu \mathrm{m} ;(\mathrm{E}, \mathrm{J}, \mathrm{O}): 25 \mu \mathrm{m}$.

\section{Supplemental figure legends}

Supplemental Figure 1: Hematoxylin/Eosin staining of stomach tissue for control (Ctrl) and Gata4 knockout (G4KO) embryos at E13.5 (A, B), E15.5 (C, D) and E17.5 (E, F). No morphological anomalies are observed in the stomach epithelium of E13.5 and E15.5 G4KO embryos, compared to Ctrl embryos ( $\mathrm{n}=5$ embryos analyzed for each stage and group). At E17.5, developing gastric units are well formed in control embryos 
(E) while G4KO stomach lacks gastric glands and the epithelium is flattened (F). Immunohistochemistry analyses revealed robust expression of parietal ( $\mathrm{HK}^{+}$-ATPase) and $\mathrm{G}$ cell (Gastrin) markers in control stomach (G and I, respectively). By contrast, the G4KO embryonic stomach fails to express any of these gastric cell markers (H, J). Scale bars: $25 \mu \mathrm{m}$

\section{Supplemental Figure 2: Up-regulation of Sonic hedgehog expression in Gata4KO} antrum and normal $\mathrm{Ngn3}$ and Hes 1 expression in the pancreas and stomach of Gata4KO embryos. Immunofluorescence analysis using Sonic hedgehog (SHH) antibody (goat anti-Shh (R\&D Systems, AF445) show strong GATA4 expression but low SHH expression in E17.5 control antrum (Ctrl) (A-C). By contrast increased SHH expression is observed in the antrum of Gata4 $\mathrm{KO}(\mathrm{G} 4 \mathrm{KO})$ embryos (D-F). Of note, SHH is absent in the ectopic pancreas (ep). Dissected pancreata and stomach from three E13.5 or E15.5 embryos of control (Ctrl) or Gata4KO (G4KO) embryos were pooled to obtain RNA. Expression of Ngn3 and Hes 1 was examined by quantitative RT-PCR in three different samples of stomach $(\mathrm{G})$ and dorsal pancreas $(\mathrm{H})$ at E13.5 and E15.5 control (Ctrl) and Gata4 knockout (G4KO) embryos (G). Total RNA isolation and qPCR was performed as previously described (Carrasco et al., Journal of Clinical Investigation, 2012, 112, 3504-3515), using the following Taqman probes (Applied Biosystems): Ngn3: Mm_00437606; Hes1: Mm_01342805_m1; $\beta$-actin: Mm02619580_g1. Gene expression data are normalized to $\beta$-actin mRNA levels and presented as mean \pm SEM. Immunofluorescence analysis of E-cadherin and smooth muscle $\alpha$ actin (I) shows normal morphology of the dorsal pancreatic epithelium of E13.5 G4KO embryos without contact with the glandular stomach epithelium, similar 


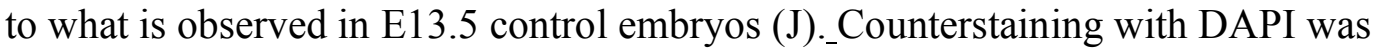
performed to visualize nuclei (blue). Scale bars: (A-F): $25 \mu \mathrm{m}$; (H, I) $100 \mu \mathrm{m}$.

Supplemental Figure 3: Normal glandular stomach morphology in Gata $^{\text {flox/flox }}$; Ptfla-Cre mice. Hematoxylin/eosin staining reveals similar morphology of the glandular stomach in control (A) and Gata4 floxflox; Ptfla-Cre mutant mice (B) at P1. Immunohistochemical analyses show strong GATA4 (C, D) and $\mathrm{HK}^{+}$-ATPase (E, F) expression in the glandular stomach of both control and Gata4 floxflox; Ptfla-Cre P1 neonatal mice. 


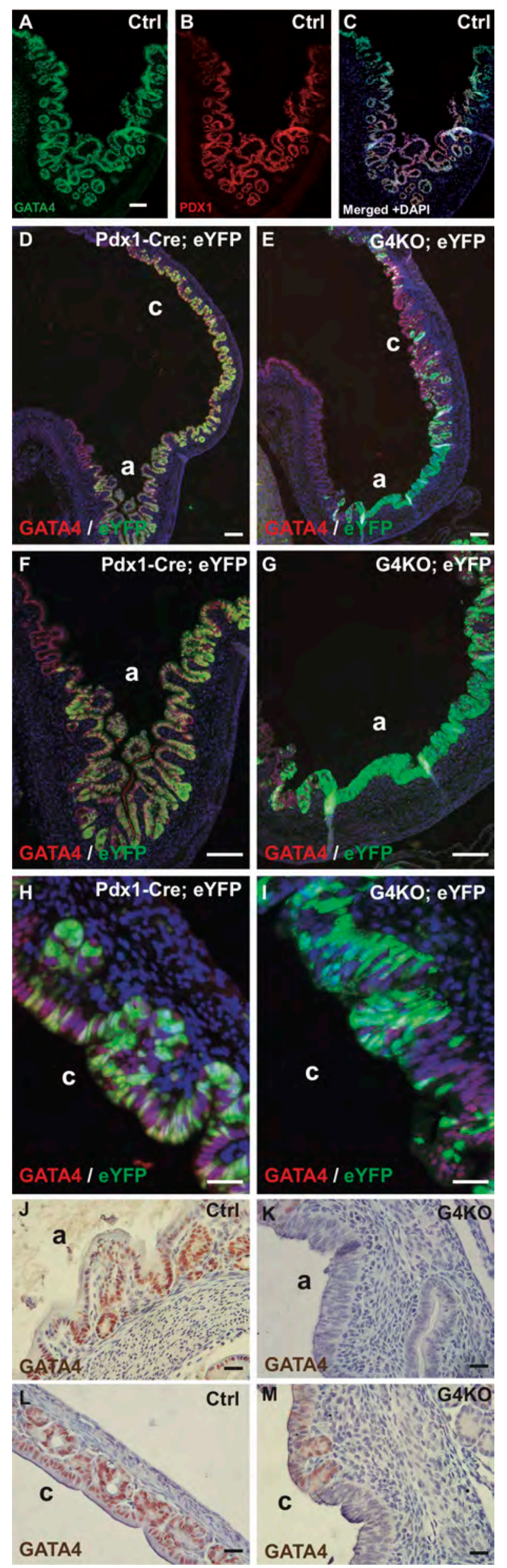


P1

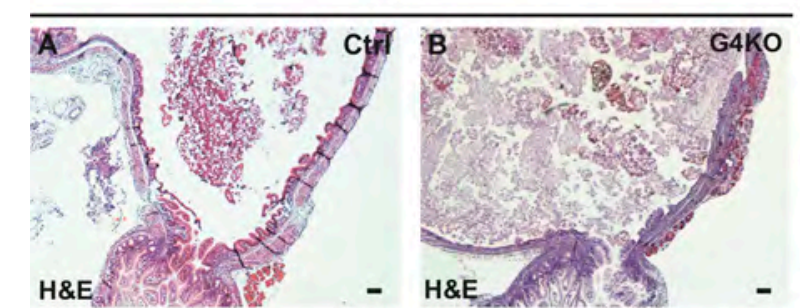

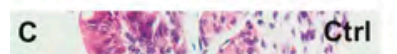

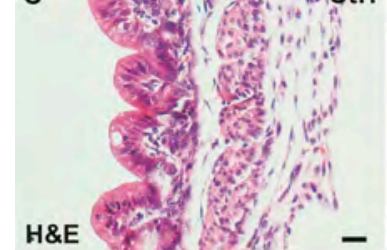

I. Ctri
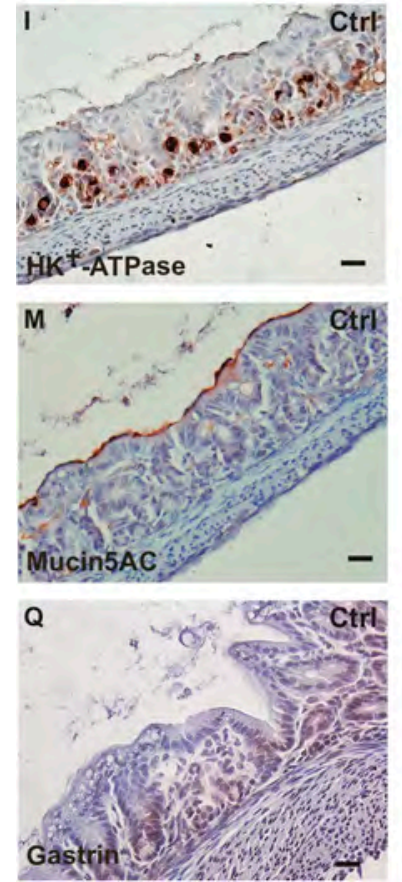
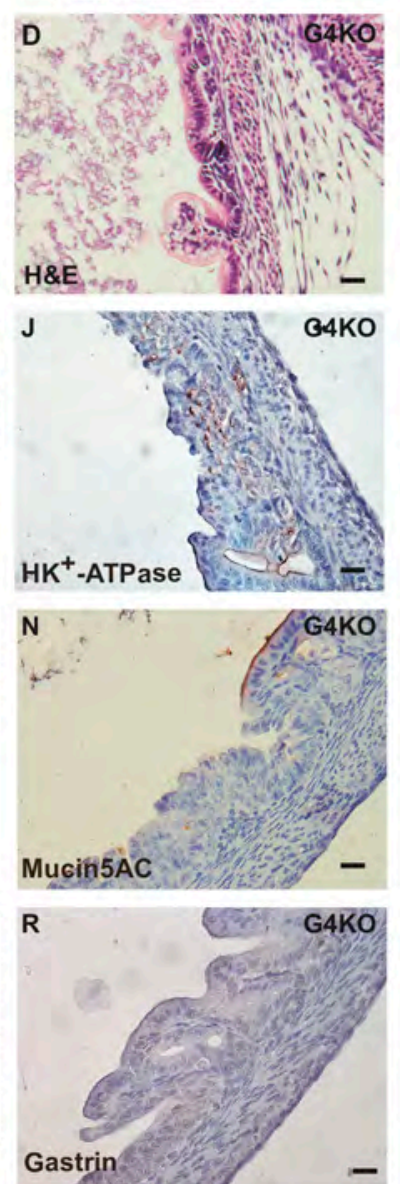
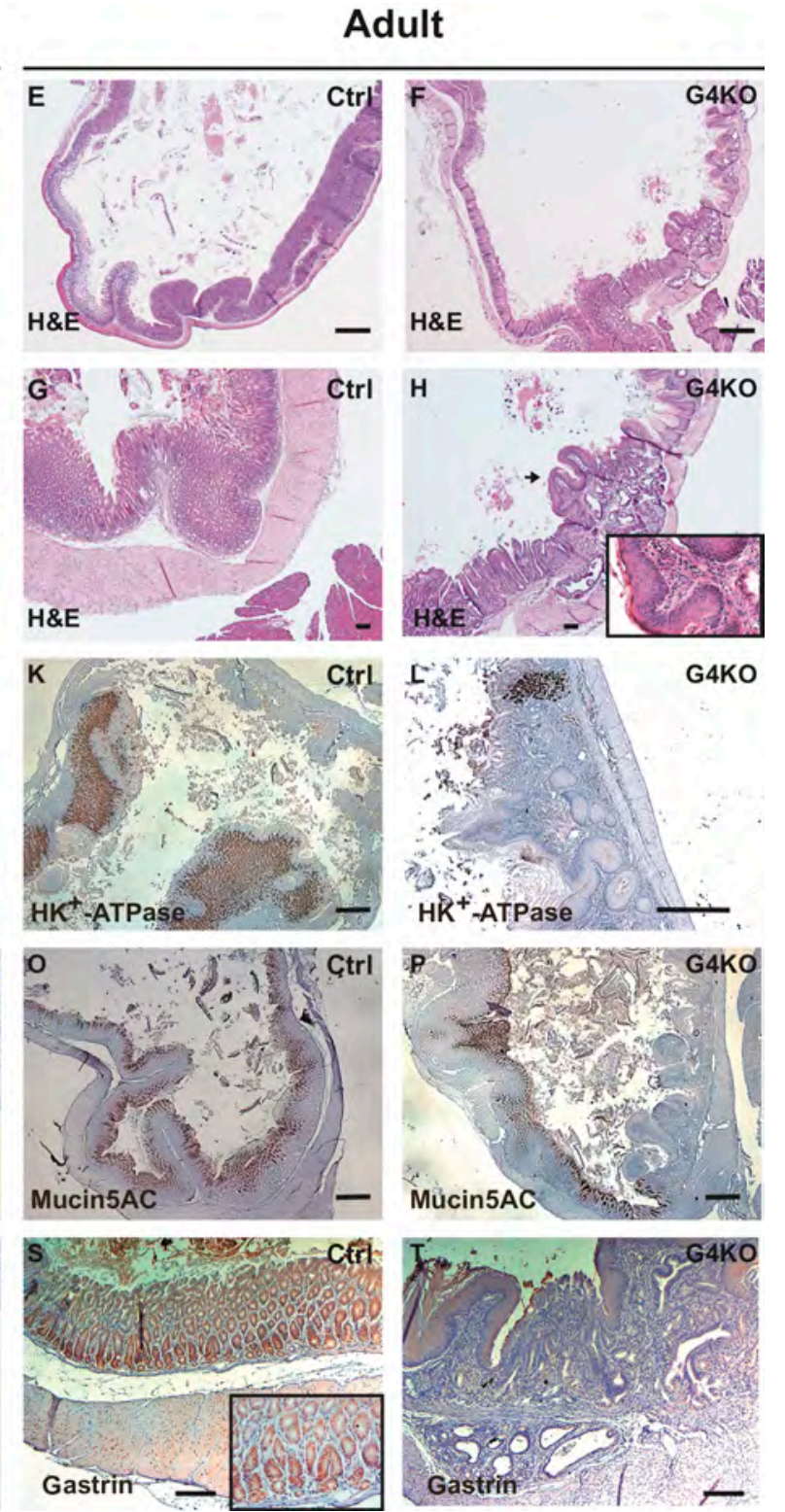
Krt14
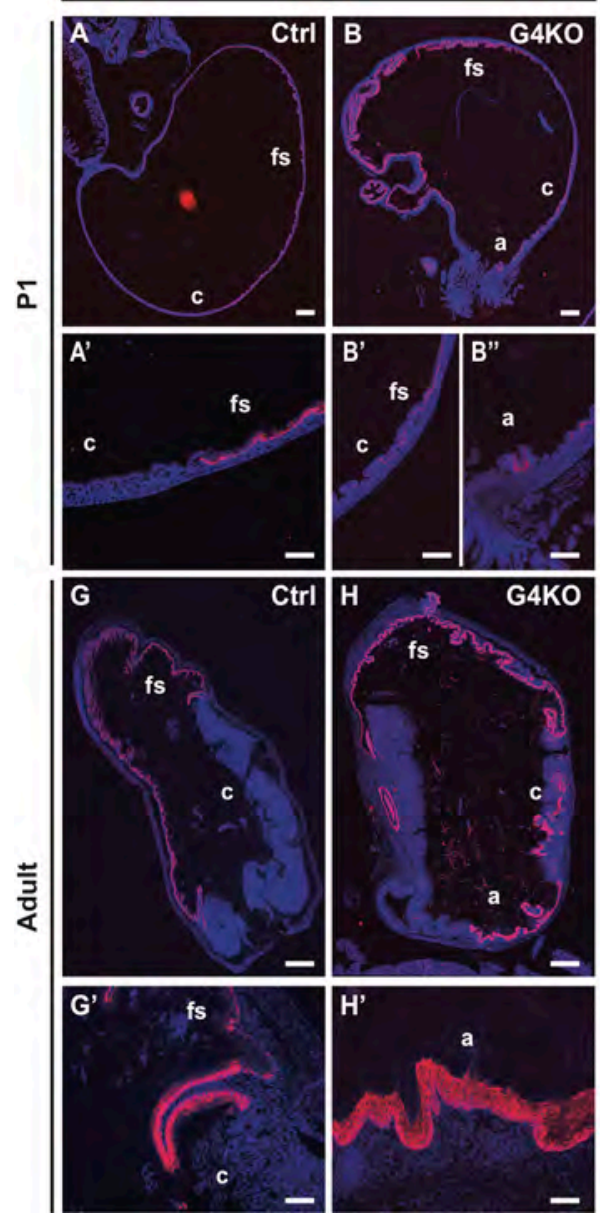

GATA4/TRP63
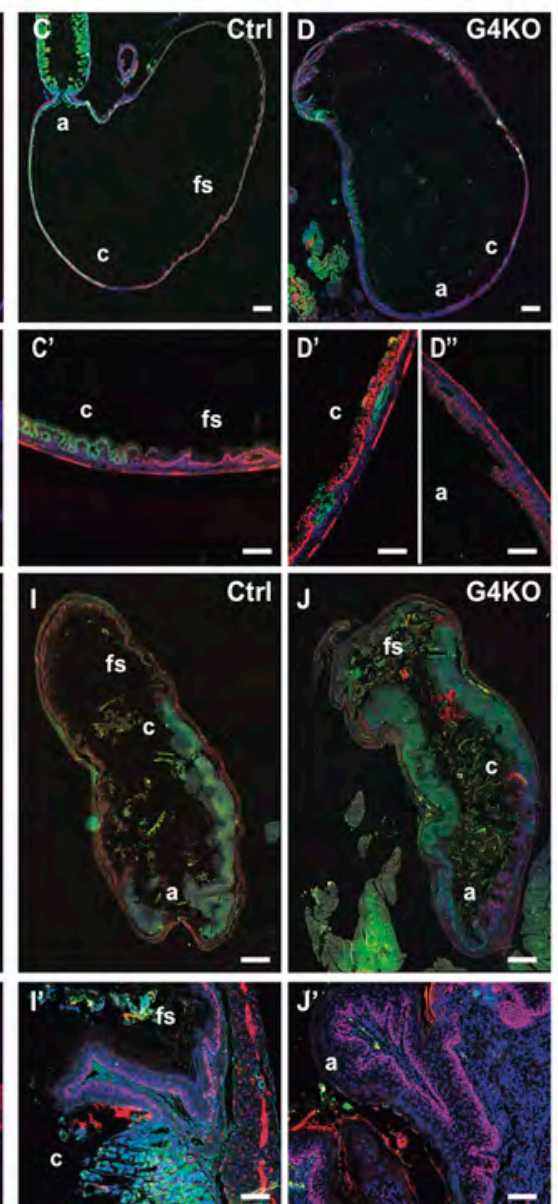

GATA4/SOX2
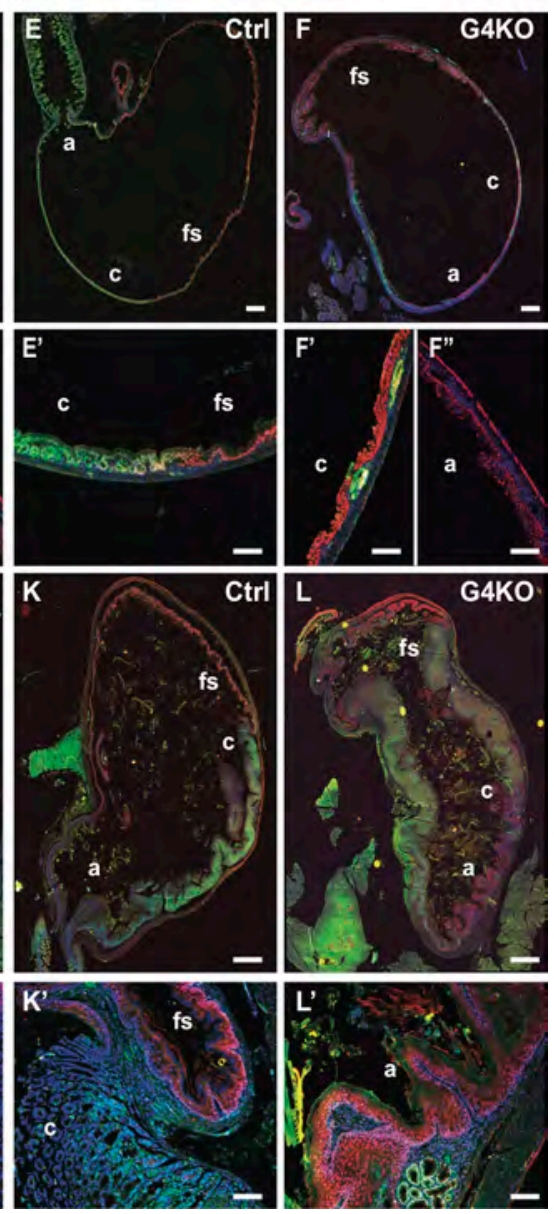

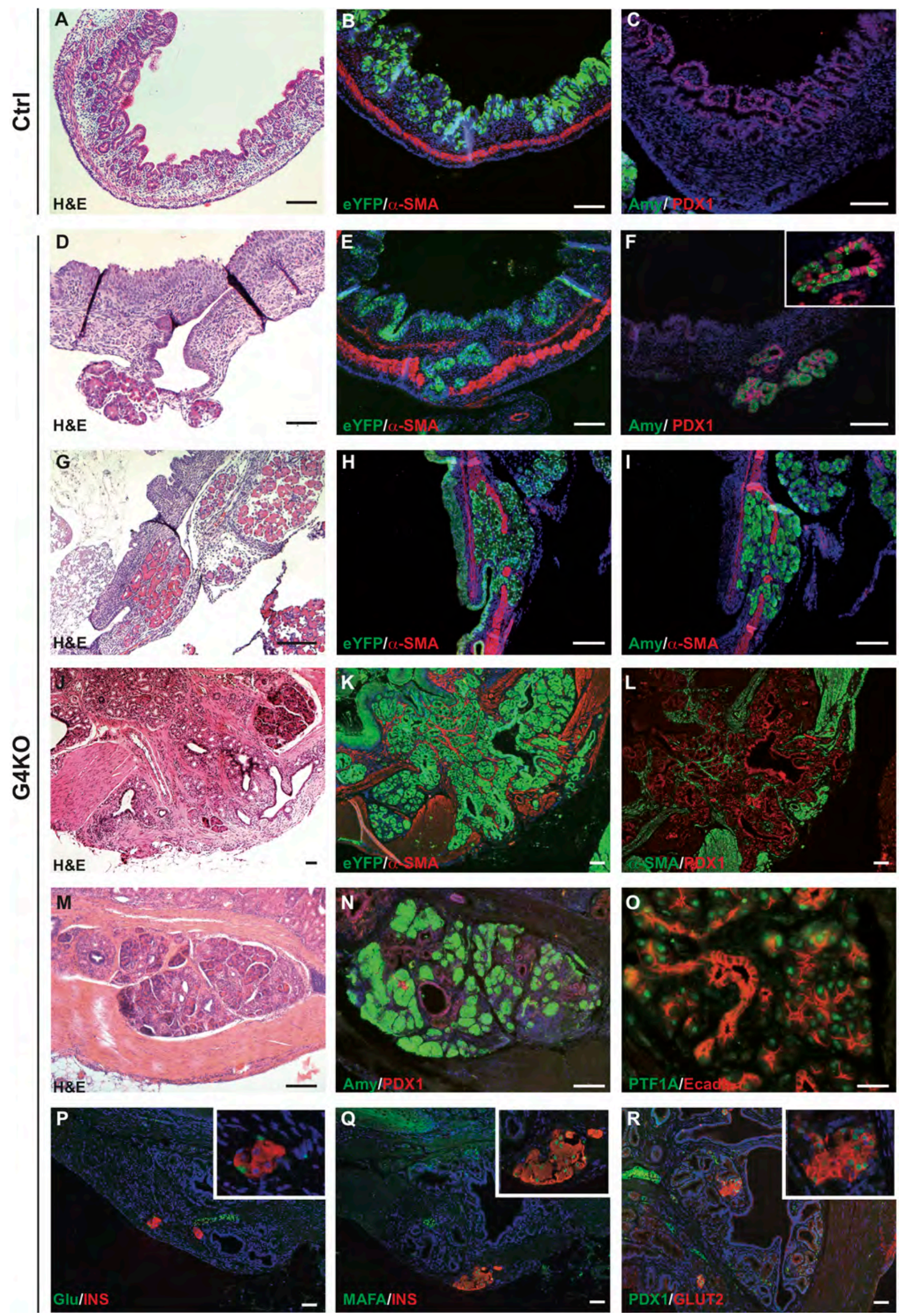
Ctrl
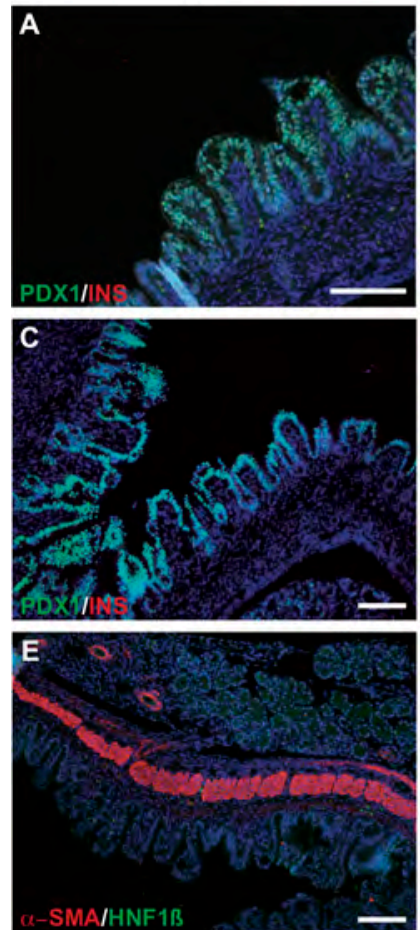

G

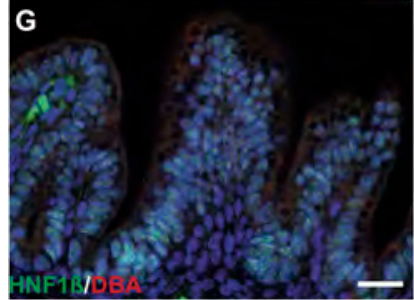

G4KO
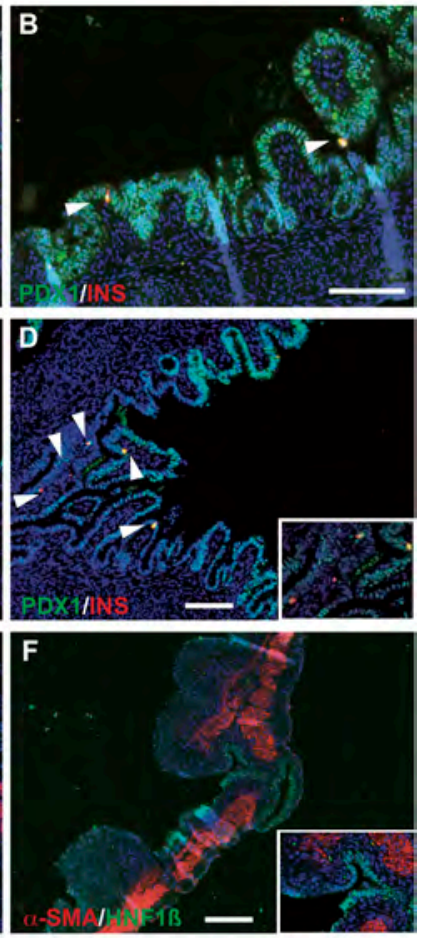

H

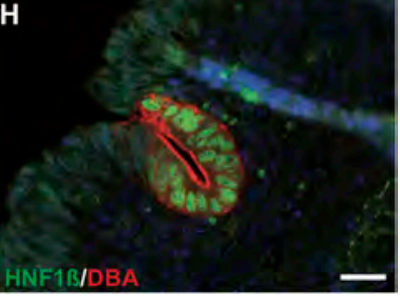



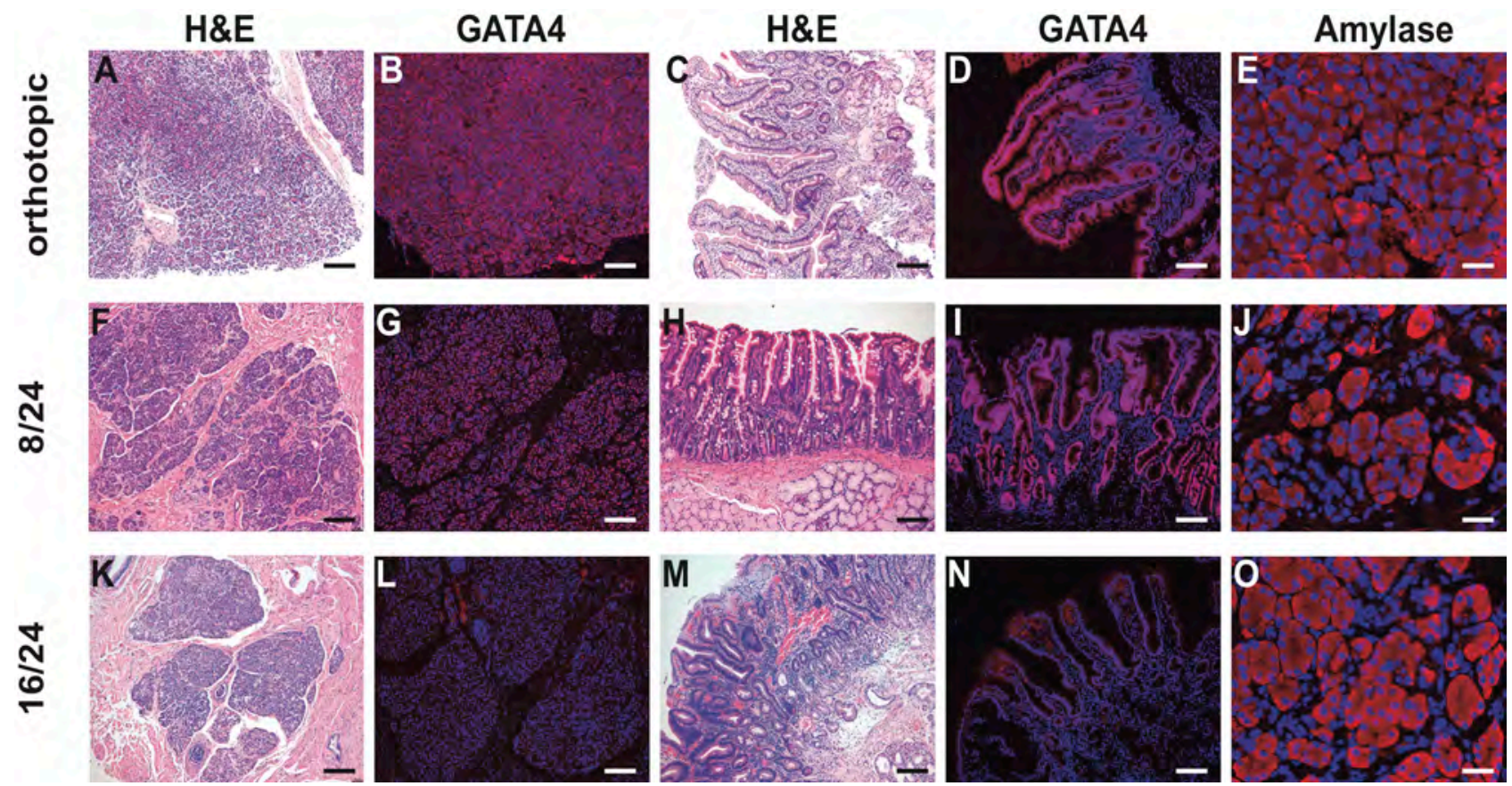